\title{
Introduction to SNPs: Discovery of Markers for Disease
}

\author{
Michael P. Weiner ${ }^{1}$ and Thomas J. Hudson ${ }^{2}$ \\ 1454 Corporation, Branford, CT, USA, and 2 Montreal Genome Centre, \\ McGill University Health Centre, Montreal, Quebec, Canada
}

BioTechniques 32: S4-S13 (June 2002)

\section{INTRODUCTION}

The molecular structure of DNA as we know it today was first proposed in 1953 in a landmark paper by Watson and Crick (56). Thirty years later the Human Genome Project (HGP) was first conceived as a joint effort between the Department of Energy (DOE) and the National Institutes of Health $(\mathrm{NIH})$ in a research plan published in 1993 and updated in 1998 (6; http://www.nhgri.nih.gov/HGP/HGP_goals/5yrplan.html) to determine the human genome sequence. At that time it was projected to be a 15-year project (1990-2005) at a cost of $\$ 200$ million per year (for a total cost then estimated at approximately $\$ 3$ billion). A little over 9 years later, the first working draft of the human genome sequence was announced at the White House on June 26, 2000. Work continued to be done on annotating the sequence, with publication of two draft versions (one private, the other publicly funded) of the sequence occurring on February 2001 (23,55). Fifty years after the structure became known, and 10 years after the start of the HGP, we now have a publicly supported human DNA sequence with over 95\% completion deposited in the public database known as Genbank (2; see also http://www.ncbi.nlm.nih.gov/Genbank/ GenbankOverview.html).

And as the scientific community anticipated the final completion of the DNA sequence of an entire representative human genome, much attention became focused on single nucleotide polymorphisms (SNPs), the most prevalent form of genetic variation. A SNP is estimated to occur approximately every one thousand base pairs or less, with a total of over ten million in the human genome (7). Due to their frequency and distribution, SNPs may serve as superior genetic markers for assembly of a high-resolution map, aiding in the identification of disease-related loci $(30,31,37,46)$. So while the private and publicly sponsored sequencing effort neared completion a few years ago, a separate, privately financed (but publicly available) effort was initiated to make the HGP more usable to researchers involved in studying human genetics. This new effort evolved into The SNP Consortium (an acronym within an acronym that became known more simply to most researchers in the genomics field as the TSC) (24; http://snp.cshl.org/); a two-year multimillion dollar nonprofit project to map human SNPs and create a database that would be made available to the public (35). This orga- nization of eleven major pharmaceutical and technology companies, four major genomics centers, and the Wellcome Trust eventually mapped close to a million SNPs by the end of 2001 .

The public and private genome efforts have given us the parts list (i.e., the DNA sequence) with which to study. But it's the inter-connectivity and working relationships of the parts that now need to be determined. And so just as every new beginning comes from some other beginning's end, we are ending the era of determining the sequence of the genetic code and entering the beginning of the age of deciphering the biology of life underlying that code. Both the Genbank and TSC databases have become important resources to investigators studying genetics and genomics. However, more efficient and cost-effective technologies still need to be developed to fully exploit these databases.

This supplement was begun as an attempt to bring together a small collection of articles on several of the SNP and haplotyping tools that are being used for this "deciphering of life." We somewhat artificially constrained the supplement in scope to tools that are being used in rather high-throughput procedures. The supplement was not meant to be a review of all technology being used for SNP and high-throughput SNP analysis. Several excellent reviews on this topic already exist, and the reader is greatly encouraged to examine them and the references contained therein (see for example References 18, 27, 28, 29, 51). In addition, this supplement was not meant to be a lexicon of genetic terminology. An excellent lexicon has already been written (10) and another one is available on the web at http://www.genomicglossaries.com/.

Rather, what we have tried to do is assemble a collection of articles that describe the use of SNPs and haplotyping in pharmacogenomics, and some technologies (for example, the article on whole genome amplification, as demonstrated in the work of John Nelson, and the article on primer design by Ray Miller, both within this Supplement) that have been brought forth to study them. We have tried to include a representation of both platform technologies and assays (see Figure 1). Current assays, loosely defined as the format used to generate the signal for SNP detection and screening include restriction fragment length polymorphism (RFLP) analysis, single-strand conformation polymorphism analysis (SSCP) (43), allele-specific oligonucleotide hybridization (ASO) (49), oligonucleotide ligation assay (OLA) (32), primer extension or mini-sequencing type assays (53; P. Bell et al., this Supplement), TaqMan ${ }^{\circledR}$ (34; F.M. De 
la Vega et al., this Supplement), molecular beacons (54) nuclease- (16) and structure-specific nuclease invader technology $(13,40 ;$ M. de Arruda, this Supplement). The actual read-out signal resulting from the assay can depend on the format of the assay and the type of platform used. The read-out can be of several types: radioactive, fluorescent, chemiluminescent, enzymatic, an analysis of the resulting product size, charge, or mass, and so forth. A quick scan of the literature reveals that many more types of assays and read-out technologies are being developed all the time to overcome both real and perceived shortcomings to existing formats.

The assays themselves can often be performed on one or several different types of platforms. A platform in this case loosely being defined as the piece of equipment on which the assay is analyzed. A variety of platforms have been used to quantify or measure reaction products. These include, for example, gel electrophoresis, capillary electrophoresis, fluorescence polarization scanners (5), semiconductor chips (15), mass spectrometry (14; C. Rodi et al., this Supplement), instruments that measure changes in redox potentials, and flow cytometry $(4,22,42)$ where the signal is flowed past the detector, and an analysis using beads wherein the detector is moved and the beads are held stationary (A. Oliphant et al., this Supplement).

Most assays and platforms start off with genomic DNA and require some form of amplification step at the front end. In general, amplification is accomplished using PCR, although other methods of amplification [including Invader ${ }^{\mathrm{TM}}$ (13), and rolling circle amplification (12), etc.] are in common use. Generally amplification is done in a uniplex format, although methods for routinely multiplexing the amplification step are beginning to become more reliable. And in some methods, the appearance of the amplification product itself is the assay (see the article by $\mathrm{M}$. Arruda, this Supplement).

\section{INCREASED THROUGHPUT AND AUTOMATION}

Throughput can be increased through the use of miniaturization, multiplex analysis, and automation. To reduce cost and increase throughput, many researchers (both public and commercial) have tried to miniaturize many of these technologies (P. Bell, this Supplement). Miniaturization often reduces reagent cost, but often makes the associated equipment more expensive, since most common laboratory equipment is not made to handle the reduced volumes being used in these miniaturized systems.

An alternative to miniaturization (and sometimes used in conjunction with) is to make the assays more parallel, often also referred to as more "multiplex." As an example, Affymetrix's chip technology uses miniaturized, high-density arrays of oligonucleotide probes on silicon wafers. Once fabricated, these probe arrays are ready for hybridization. The nucleic acids to be analyzed (i.e., the target) are labeled with a fluorescent reporter group. The labeled target is then incubated with the array using a fluidics workstation. After the hybridization reaction is complete, the array is inserted into a fluorescent scanner, where patterns of hybridization are detected. The hybridization data are collected as light emitted from the fluorescent reporter groups incorporated into the target, which is now bound to the probe

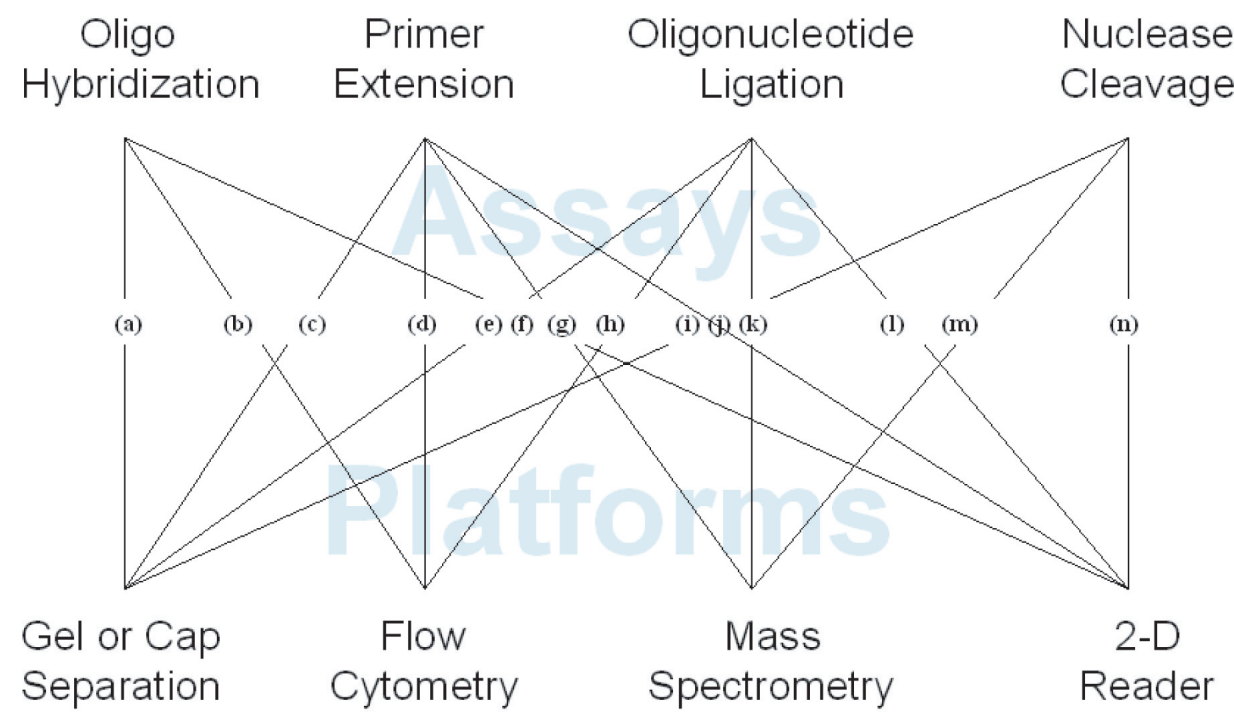

Figure 1. Two dimensional representation of possible mix and matches of SNP genotyping assays and platforms [Adapted from Ivo Gut (18)]. Various read-out methods (for example, radioactive, fluorescent, chemiluminescent, flourescence energy transfer, fluorescent polarization, etc.) can be used to represent a potential third dimension. Examples for use of these assays on a particular platform can be found in references and commercial Web sites for products as follows: (a) 51 , (b) 1 , (c) 36, (d) 57 (e) 41, (f) 8, (g) 50, (h) 22, 42 (i) 3, (j) 11, (k) 33, (l) 17, (m) 52, (n) 13. 
array. Probes that perfectly match the target generally produce stronger signals than those that have mismatches. Since the sequence and position of each probe on the array are known, the identity of the target nucleic acid applied to the array can be determined. By doing this in a highly parallel fashion, they thus allow the costs of supplies and reagents to drop dramatically.

Another way to multiplex is through temporal separation, such as can be performed with electrophoretic or mass spectrometric analysis of mixtures of molecules with varying sizes or charges. Another example of temporal separation is the use of flourescently distinguishable beads in flow cytometry $(4,22,42)$. One of us (M.P.W.) has previously developed a high-throughput SNP analysis assay utilizing an array of differentially fluorescent microspheres (see Figure 2). The array of microspheres coupled with identifying sequences, termed complementary ZipCodes (cZipCodes; see Figures 2 and 3) allow for multiplexing possibilities. For one of two given assays developed [the single base chain extension (SBCE) format; see also the article by P. Bell et al., this Supplement] the querying of a polymorphic base involves extending an oligonucleotide containing a ZipCode sequence and a SNP-specific sequence with a DNA polymerase and fluoresceinated dideoxynucleotides (see Figure 3). In a second assay developed (the OLA format) the querying of a polymorphic base involves ligating an oligonucleotide containing a ZipCode sequence and a SNP-specific sequence with a DNA ligase and reporter-labeled oligonucleotide.

The ZipCode portion of the oligonucleotide hybridizes with the cZipCode covalently attached to a substrate. The substrate can be a chip, glass slide, or, as in the method developed, a microsphere. In addition to multiplexing capabilites, ZipCode systems allow multiple sets of SNPs to be analyzed by a limited set of cZipCode attached substrates. A concern with such highly parallel analysis, however, is the risk that some of the targets and capture probes will cross-hybridize. When empirically tested, this resulted in about $15 \%$ cross- and weak-hybridization of ZipCodes in conditions used for a bead-based assay (see Figure 4). These ZipCodes are typically discarded.

Other highly multiplexed formats have been developed for mass spectroscopy (26) and can be found in discussions by Lechner et al. (33). In the Kokoris (26) system for SNP genotyping, a unique family of low-molecular-weight tags are conjugated to oligonucleotides via a photolabile linker. Each tag has a different mass that is designated for a specific oligonucleotide sequence. SNP-specific primers and universal masscode tagged primers are used in a PCR-based SNP-discrimination assay. SNP-specific PCR products are purified to remove unincorporated primers and the tags are cleaved from the purified PCR products by exposure to a 254 -nm mercury lamp and then injected into a single quadrupole mass spectrometer, which detects the weight of each tag species. The raw analytical data from the mass spectrometer is converted into a genotype call. In the current format, 30 tags can be used to multiplex 15 SNPs per well, with a throughput capacity of more than 40000 genotypes per mass spectrometer per day (26).

Automation in SNP analysis has been greatly facilitated through the use of liquid handling robots, plate movers, various kinds of "hotels," incubators, and rails. The laboratory bench has now been exchanged for tables and platforms supporting rail systems supplied by new and traditional equipment manufacturers.

\section{RATIONALE FOR HAPLOTYPE MAPPING}

Numerous empirical studies have shown that nearby SNP alleles are often in linkage disequilibrium (LD) with each other, such that the state of one SNP allele is often highly correlated with the allele of another close SNP. These correlations exist because of the shared history of tightly linked SNPs — which are

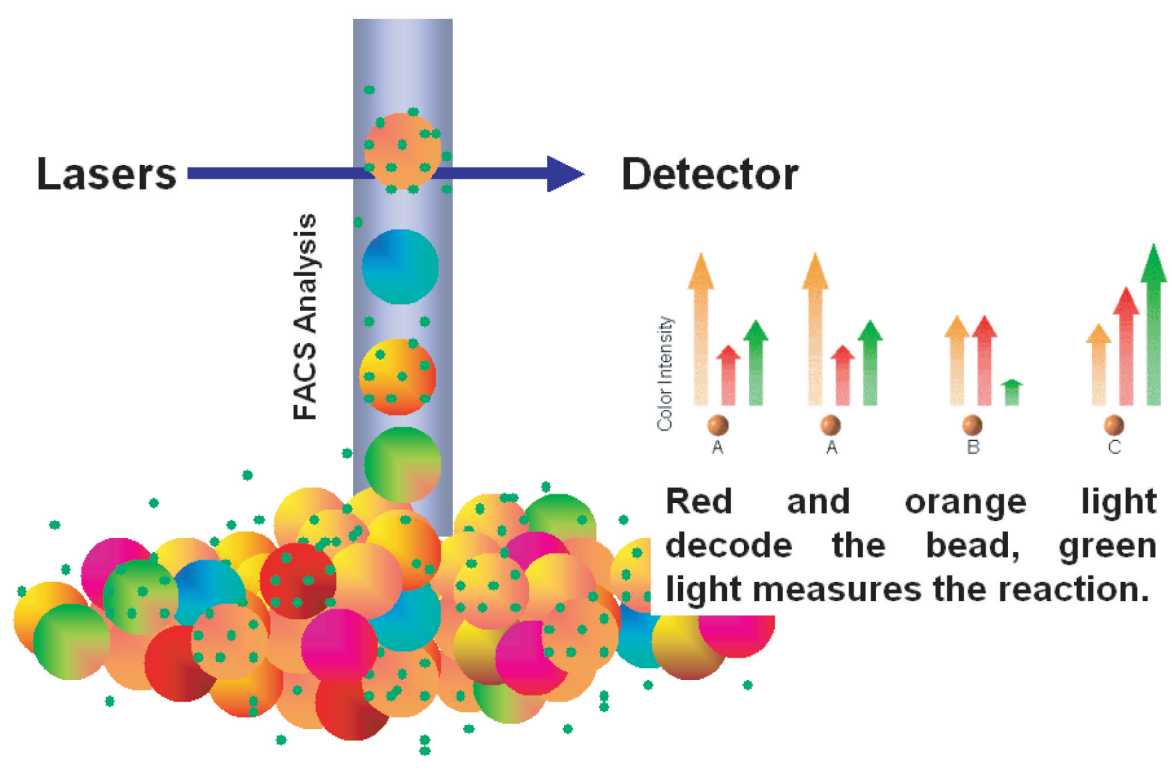

Figure 2. Use of beads for multiplex analysis. See text and References 22 and 42 for details. 


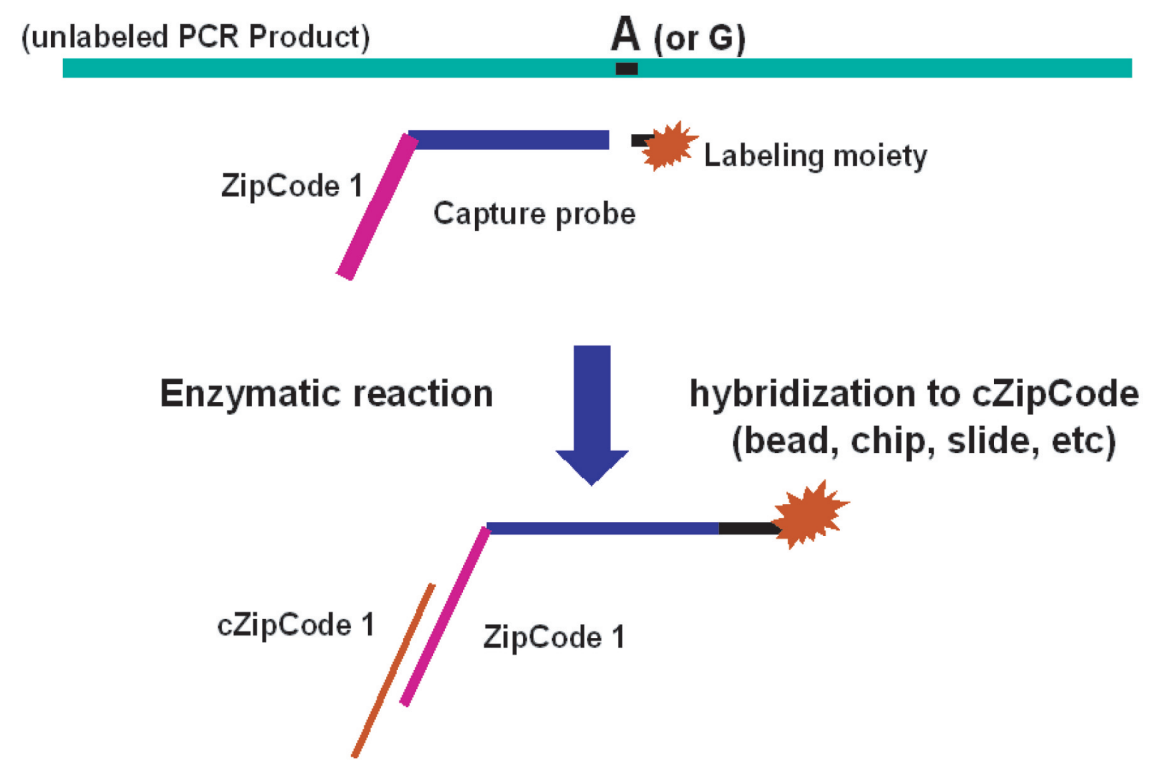

\section{Solid Phase Substrate}

(bead, chip, slide, etc)

\section{Analysis}

Figure 3. ZipCoded SNP analysis. Capture-probe oligonucleotides are designed containing a $3^{\prime}$ region complementary to a region surrounding the SNP being queried, and a $5^{\prime}$ region encoding a complementary ZipCoded region. An enzymatic step is used to add a label to the capture probe, and the ZipCode, via hybridization, is used to read the presence of the signal on a bead, chip, slide, etc.

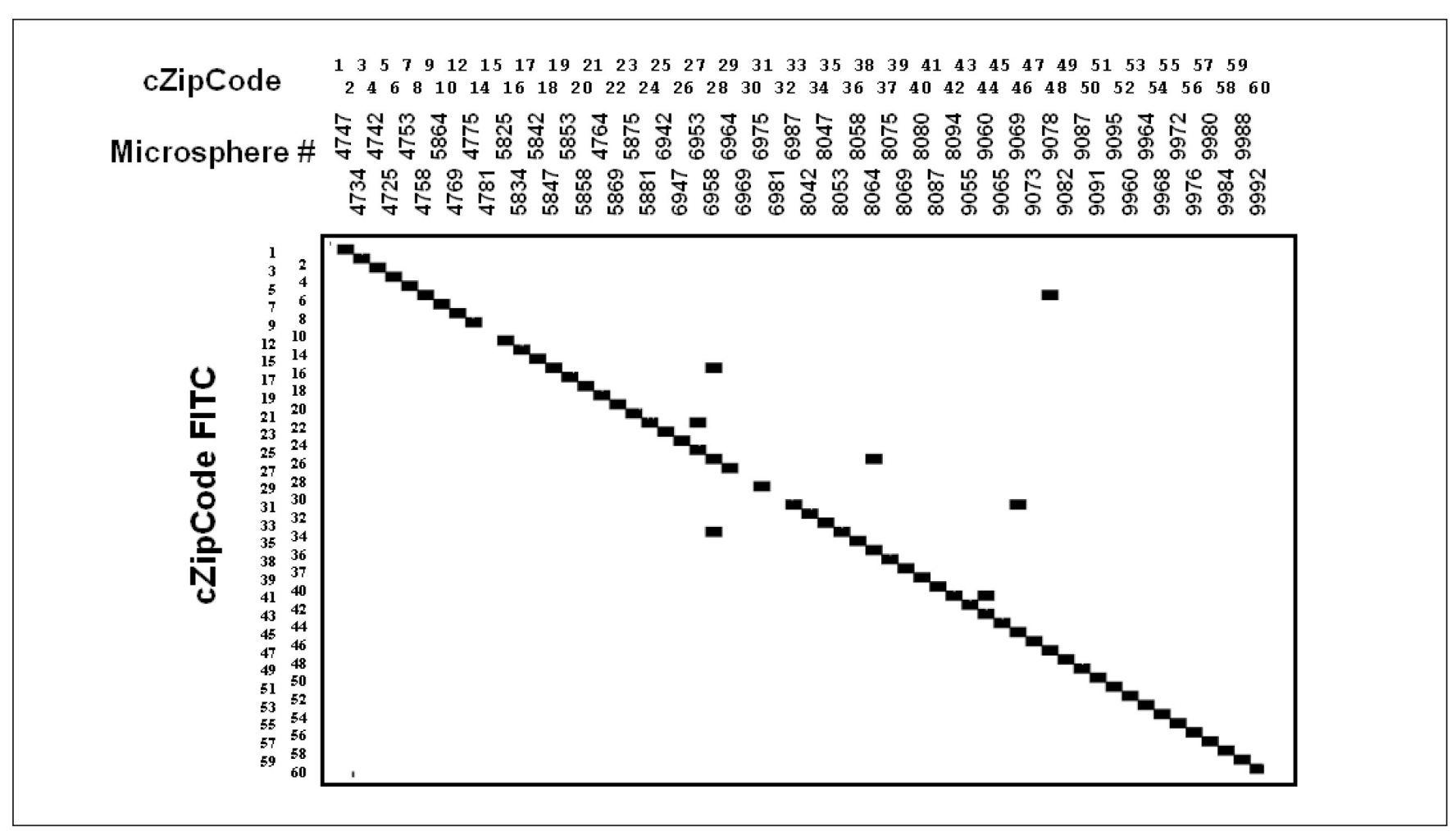

Figure 4. Validation of ZipCodes. ZipCodes need to be empirically tested under the conditions used for hybridization. Although a computer algorithm was first used to analyze the 60 ZipCodes, approximately 15\% still either cross-hybridized or yielded substandard hybridization signal (see also References 22, 42, and 57). 
co-transmitted from generation to generation. Patterns of human sequence variation (haplotypes) thus represent ancestral DNA segments. Historical meioses have slowly dissociated alleles from neighboring alleles on ancestral chromosomes, except for tightly linked variants. The extent of linkage disequilibrium had been the object of numerous studies, particularly in the cloning of simple Mendelian disorders such as cystic fibrosis (25), Huntington's disease (21), and diastrophic dysplasia (DTD) (19). Whereas these cloning studies benefited from the large chromosomal segments showing LD spanning over large distances (sometimes in the megabase range), very little empirical data was available until recently regarding $\mathrm{LD}$ across the human genome in the world population.

We focus on three recent examples of large-scale surveys of LD (and haplotypes):

1. Reich et al. (45) sampled 19 chromosome regions for their SNP content. High-frequency SNPs spanning intervals of 2 to $160 \mathrm{~kb}$ were first genotyped in Caucasian samples. Over all regions, $\mathrm{LD}$ was detectable at distances of about $60 \mathrm{~kb}$, with a significant difference between regions, as the range was as short as $6 \mathrm{~kb}$ at one locus and as long $155 \mathrm{~kb}$ in another. Not surprisingly, LD was significantly correlated with the estimated local recombination rates. Further analysis in a Nigerian sample provided evidence of shorter $\mathrm{LD}$ in this population, although the allelic combinations over short distances were similar to the Caucasian sample. Overall, this work provided evidence that large blocks of LD are common across the human genome, and that genome-wide LD mapping of disease genes will be feasible.

2. Rioux $(47,48)$ and Daly (9) defined the haplotype structure at the IBD5 locus using high-density SNP analysis. Strong $\mathrm{LD}$ signals, as well as a common haplotype were observed in patients with inflammatory bowel disease (IBD). The project involved a hierarchical approach to LD mapping, where the IBD5 locus (47) was narrowed down from an 18-cM linkage peak to a common disease haplotype spanning $250 \mathrm{~kb}$. Over 100 SNPs (with greater than $5 \%$ minor allele frequency) in a $500-\mathrm{kb}$ region were used to define a high-resolution haplotype structure, which is remarkably simple: eleven chromosomal segments (called haplotype blocks) containing most of the DNA sequence are observed at this locus. The blocks vary in size from 3 to 92

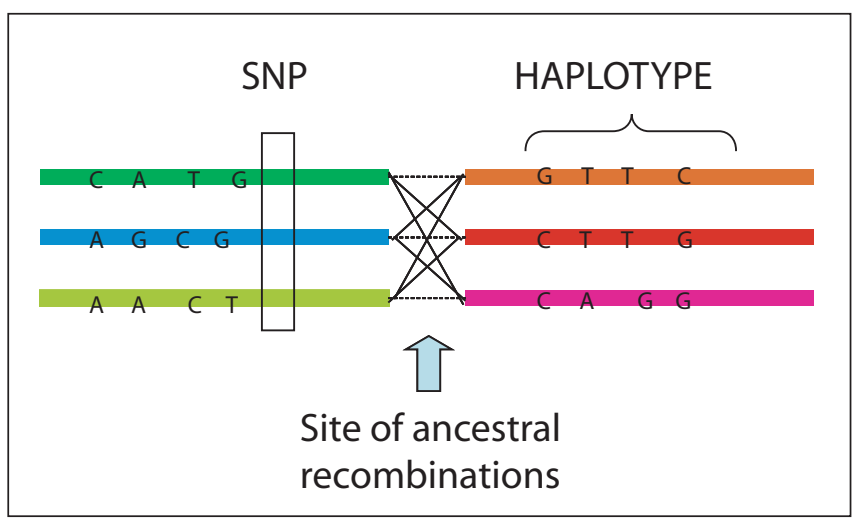

Figure 5. Block-like haplotype diversity. Stretches of continuous DNA containing a distinctive set of alleles are called haplotypes. Block-like haplotype diversity refers to the observation of DNA segments with a limited number of common haplotypes separated by recombinational hotspots. kb. Each block is defined by only two, three, or four common haplotypes. Suggestive of hotspots, the blocks are separated by apparent sites of ancestral recombination. There is significant correlation between blocks, giving rise to long-range LD. In this project, haplotype analysis proved to be more robust than with the more noisy single SNP analysis. More importantly, the findings suggest that whole-genome studies will be simpler than previously thought - as haplotype information may be deduced by sampling only a subset of SNPs (see Figure 5).

3. Patil et al. (44) confirmed the large-scale existence of haplotype blocks through a comprehensive study of human chromosome 21 . They used high-density oligonucleotide arrays, in combination with somatic cell genetics, to identify a large fraction of all common human chromosome 21 SNPs. This allowed the direct observation of the haplotype structure across the chromosome. The project revealed blocks of limited haplotype diversity, generally characterized by only three common haplotypes in more than $80 \%$ of tested chromosomes. The majority of the haplotype information for an entire chromosome could be captured by the selection of two or three SNPs per block of SNPs.

The implications of these findings are significant for human genetic and pharmacogenetic studies. The haplotype block structure implies that it will not be necessary to test the $>10000000$ common SNPs in future genome-wide association mapping projects. Future disease association studies will focus on genotyping only a handful of SNPs per haplotype block, to extract the information needed to detect a disease variant within the block.

The momentum is growing to generate a Haplotype Map of the Human Genome (20), to provide a tool for systematic testing of the entire genome for genes that predispose to common diseases such as asthma, diabetes, hypertension, and so forth. These projects strongly support the need to foster further development of new high-throughput technologies for genome-wide genetic studies.

\section{CONCLUSIONS}

For SNPs to be useful in studying human diseases, there are several requirements for success. These include bioinformatics (an example screen dump is shown in Figure 6) and statistical analysis tools, access to patient resources, and new genetic technologies. The massive amounts of data that genomic studies generate are not knowledge; bioinformatics and statistics will be needed to analyze these data and convert this information to knowledge. The potential utility of SNPs to help establish individualized medicine is enormously exciting. The powerful, target-specific pharmaceuticals being developed today can bring profound improvements to the lives of many patients, but may have serious side effects in certain subpopulations. The genetic variation behind these differing biological responses may correlate with a small set of SNPs that could serve as a diagnostic tool to ensure the prescription of "the right medicine to the right patient."

\section{ACKNOWLEDGMENTS}

New technologies bring revolutions. The risks that others have taken to enable these SNP technologies allow for the im- 


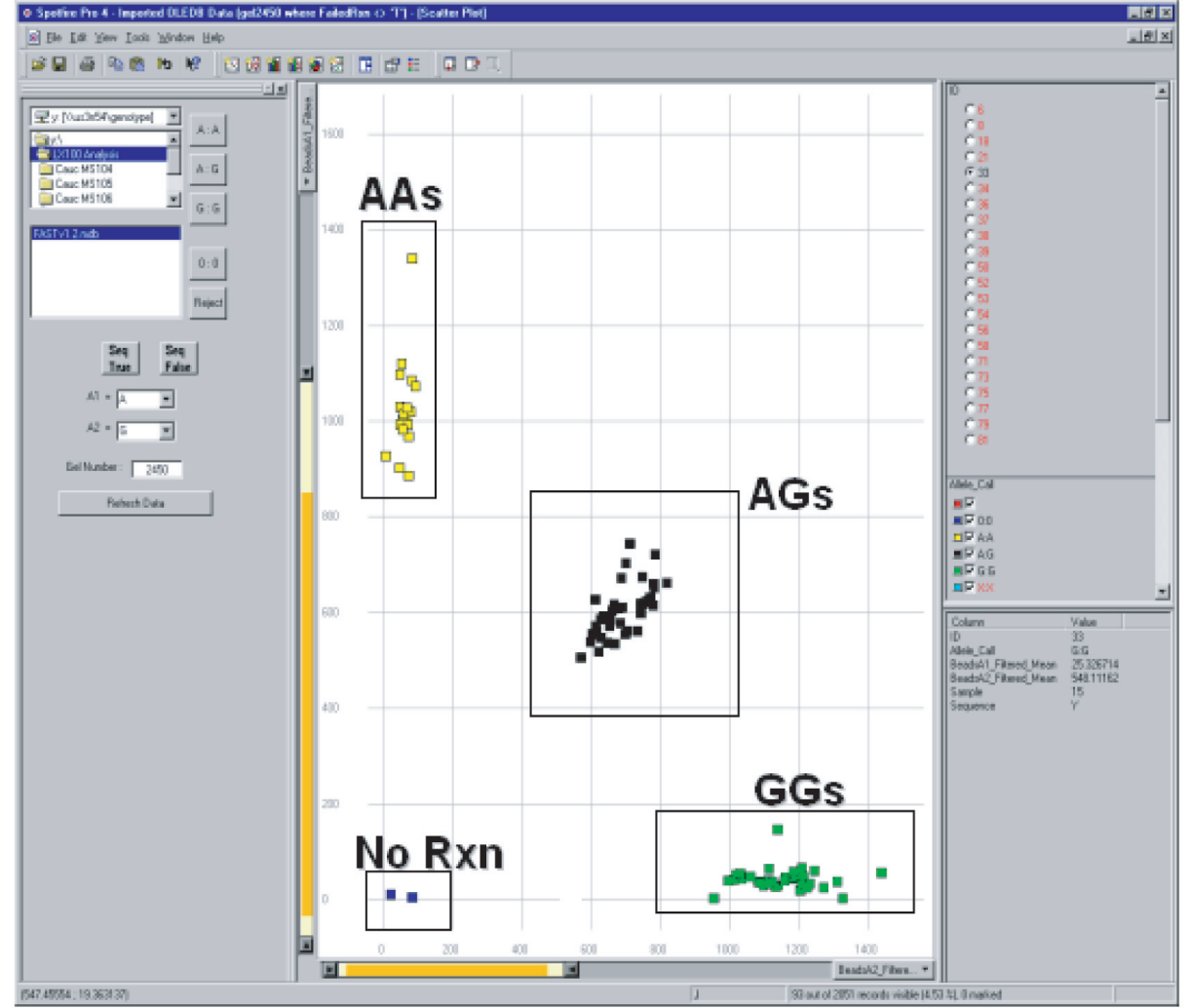

Figure 6. Data visualization tools. Spotfire or similar-type data visualization tools are used to analyze data. In fluorescent assays, heterozygote values are typically found on a diagonal line between the two homozygotes. In end-point enzymatic assays, the heterozygotes are usually found at a value equal to both homozygotes.

provements in healthcare that will benefit a great many of us. We hope that the topics covered in this supplement are able to serve as an informational starting-point to novices as well as those experienced in the area. We would like to thank Christine Emerton at BioTechniques and her team at Eaton Publishing for both initiating this supplement series to address important issues arising in the field of biology and in shepherding this particular project through the editorial process. Due to space limitations, only selected references were cited in this introduction. Our apologies go to those colleagues whose works were not cited. Where possible, we have referenced recent publications and review articles; the reader is encouraged to investigate the original research reports for experimental details.

Finally, we would like to dedicate this supplement to those individuals with unmet medical needs and to the researchers who toil each and every day to try to meet those needs with novel and better medicines.

\section{REFERENCES}

1.Armstrong, B., M. Stewart, and A. Mazumder. 2000. Suspension arrays for high throughput, multiplexed single nucleotide polymorphism genotyping. Cytometry 40:102-108.

2.Benson, D.A., I. Karsch-Mizrachi, D.J. Lipman, J. Ostell, B.A. Rapp, and

D.L. Wheeler. 2000. Genbank. Nucleic Acids Res. 28:15-18.

3.Breen, G., D. Harold, S. Ralston, D. Shaw, and D. St. Clair. 2000. Deter- mining SNP allele frequencies in DNA pools. BioTechniques 28:464-470.

4.Chen, J., M.A. Iannone, M.-S. Li, J.D. Taylor, P. Rivers, A.J. Nelsen, K.A. Slentz-Kesler, A. Roses, and M.P. Weiner. 2000. A microsphere-based assay for single nucleotide polymorphism analysis using single base chain extension. Genome Res. 10:549-557.

5.Chen, X.N., L. Levine, and P.Y. Kwok. 1999. Fluorescence polarization in homogeneous nucleic acid analysis. Genome Res. 9:492-498.

6.Collins, F., A. Patrinos, E. Jordan, A. Chakravarti, R. Gesteland, L. Walters, and the members of the DOE and NIH planning groups. 1998. New goals for the U.S. human genome project: 1998-2003. Science 282:682-689.

7.Cooper, D.N., B.A. Smith, H.J. Cooke, S. Niemann, and J. Schmidtke. 1985. An estimate of unique DNA sequence heterozygosity in the human genome. Hum. Genet. 69:201-205.

8.Cutler, D.J., M.E. Zwick, M.M. Carrasquillo, C.T. Yohn, K.P. Tobin, C. Kashuk, D.J. Mathews, N.A. Shah, E.E. Eichler, J.A. Warrington, and A. Chakravarti. 2001. High-throughput variation detection and genotyping using microarrays. Genome Res. 11:1913-25.

9.Daly, M.J., J. D. Rioux, S.F. Schaffner, T.J. Hudson, and E.S. Lander. 2001. High-resolution haplotype structure in the human genome. Nat. Genet. 29:229-232.

10.Drabek, J. 2001. A commented dictionary of techniques for genotyping. Electrophoresis 22:1024-1045.

11.Fan, J.B., X. Chen, M.K. Halushka, A. Berno, X. Huang, T. Ryder, R.J. Lipshutz, D.J. Lockhart, and A. Chakravarti. 2000. Parallel genotyping of human SNPs using generic high-density oligonucleotide tag arrays. Genome Res. 10:853-860.

12.Faruqi, A.F., S. Hosono, M.D. Driscoll, F.B. Dean, O. Alsmadi, R. Bandaru, G. Kumar, B. Grimwade, et al. 2001. High-throughput genotyping of single nucleotide polymorphisms with rolling circle amplification. BMC Genomics 2:4.

13.Fors, L, K.W. Lieder, S.H. Vavra, and R.W. Kwiatkowski. 2000. Large-scale 
SNP scoring from unamplified genomic DNA. Pharmacogenomics 1:219229

14.Fu, D.J., K. Tang, A. Braun, D. Reuter, B. Darnhofer-Demar, D.P. Little, M.J. O'Donnell, C.R. Cantor, and H. Koster. 1998. Sequencing exons 5 to 8 of the p53 gene by MALDI-TOF mass spectrometry. Nat. Biotechnol. 16:381-384

15.Gilles, P.N., D.J. Wu, C.B. Foster, P.J. Dillon, and S.J. Chanock. 1999. Single nucleotide polymorphic discrimination by an electronic dot blot assay on semiconductor microchips. Nat. Biotechnol. 17:365-370.

16.Goldrick, M.M. 2001. RNase cleavage-based methods for mutation/SNP detection, past and present. Hum. Mutat. 18:190-204.

17.Gunderson, K.L., X.C. Huang, M.S. Morris, R.J. Lipshutz, D.J. Lockhart, and M.S. Chee. 1998. Mutation detection by ligation to complete n-mer DNA arrays. Genome Res. 8:1142-1153.

18.Gut, I. 2001. Automation in genotyping of single nucleotide polymorphisms. Hum. Mutat. 17:475-492.

19.Hastbacka, J., A. de la Chapelle, I. Kaitila, P. Sistonen, A. Weaver, and E. Lander. 1992. Linkage disequilibrium mapping in isolated founder populations: diastrophic dysplasia in Finland. Nat. Genet. 2:204-211.

20.Helmuth, L. 2001. Genome research: map of the human genome 3.0. Science 293:583-585.

21.Huntington's Disease Collaborative Research Group. 1993. A novel gene containing a trinucleotide repeat that is expanded and unstable on Huntington's disease chromosomes. Cell 72:817-818.

22.Iannone, M.A., J.D. Taylor, J. Chen, M.-S. Li, P. Rivers, K.A. SlentzKesler, and M.P. Weiner. 2000. Multiplexed single nucleotide polymorphism genotyping by oligonucleotide ligation and flow cytometry. Cytometry 39:131-140.

23.International Human Genome Sequencing Consortium. 2001. Initial sequencing and analysis of the human genome. Nature 409:860-921.

24.International SNP Map Working Group. 2001. A map of human genome sequence variation containing 1.42 million single nucleotide polymorphisms. Nature 409:928-933.

25.Kerem, B., J.M. Rommens, J.A. Buchanan, D. Markiewicz, T.K. Cox, A. Chakravarti, M. Buchwald, and L.C. Tsui. 1989. Identification of the cystic fibrosis gene: genetic analysis. Science 245:1073-1080.

26.Kokoris, M, K. Dix, K. Moynihan, J. Mathis, B. Erwin, P. Grass, B. Hines, and A. Duesterhoeft. 2000. High-throughput SNP genotyping with the Masscode system. Mol. Diagn. 5:329-340.

27.Kristensen, V.N., D. Kelefiotis, T. Kristensen, and A.L. Borresen-Dale. 2001. High-throughput methods for detection of genetic variation. BioTechniques 30:318-326.

28.Kwok, P.-Y. 2000. High-throughput genotyping assay approaches. Pharmacogenomics 1:95-100.

29.Kwok, P.-Y. 2001. Methods for genotyping single nucleotide polymorphisms. Annu. Rev. Hum. Genet. 2:235-258.

30.Lai, E. 2001. Application of SNP technologies in medicine: lessons learned and future challenges. Genome Res. 11:927-929.

31.Lai, E., J. Riley, I. Purvis, and A. Roses. 1998. A 4-MB high-density single nucleotide polymorphism-based map around human APOE. Genomics 54:31-38.

32.Landegren, U., R. Kaiser, J. Sanders, and L. Hood. 1988. A ligase-mediated gene detection technique. Science 241:1077-1080.

33.Lechner, D., G.M. Lathrop, and I.G. Gut. 2002. Large-scale genotyping by mass spectrometry: experience, advances and obstacles. Curr. Opin. Chem. Biol. 6:31-38

34.Livak, K.J., J. Marmaro, and J.A. Todd. 1995. Towards full automated genome-wide polymorphism screening. Nat. Genet. 9:341-342.

35.Marshall, E. 1999. Drug firms to create public database of genetic mutations. Science 284:406-467.

36.Matyas, G., C. Giunta, B. Steinmann, J.P. Hossle, and R. Hellwig. 2002. Quantification of single nucleotide polymorphisms: a novel method that combines primer extension assay and capillary electrophoresis. Hum. Mutat. 19:58-68.

37.McCarthy, J.J. and R. Hilfiker. 2000. The use of single-nucleotide polymorphism maps in pharmacogenomics. Nat. Biotechnol. 18:505-508.

38.McDade, R.L. and R.J. Fulton. 1997. True multiplexed analysis by computer-enhanced flow cytometry. Med. Dev. Diag. Indust. 19:75-82.

39.McHugh, T.M. 1994. Flow microsphere immunoassay for the quantitative and simultaneous detection of multiple soluble analytes. Meth. Cell Biol. 42:575-595.

40.Mein, C.A., B.J. Barratt, M.G. Dunn, T. Siegmund, A.N. Smith, L. Espos- ito, S. Nutland, H.E. Stevens, et al. 2000. Evaluation of single nucleotide polymorphism typing with Invader on PCR amplicons and its automation. Genome Res. 10:330-343.

41.Nickerson, D.A., S.L. Taylor, S.M. Fullerton, K.M. Weiss, A.G. Clark, J.H. Stengard, V. Salomaa, E. Boerwinkle, and S.F. Sing. 2000. Sequence diversity and large-scale typing of SNPs in the human apolipoprotein E gene. Genome Res. 10:1532-1545.

42.Nolan, J.P. and L.A. Sklar. 2002. Suspension array technology: evolution of the flat-array paradigm. Trends Biotechnol. 20:9-12.

43.Orita, M., H. Iwahana, H. Kanazawa, K. Hayashi, and T. Sekiya. 1989. Detection of polymorphisms of human DNA by gel electrophoresis as single-strand conformation polymorphisms. Proc. Natl. Acad. Sci. USA 86:2766-2770.

44.Patil, N., A.J. Berno, D.A. Hinds, W.A. Barrett, J.M. Doshi , C.R. Hacker, C.R. Kautzer, D.H. Lee, et al. 2001. Blocks of limited haplotype diversity revealed by high-resolution scanning of human chromosome 21 . Science 294:1719-1723

45.Reich, D. E., M. Cargill, S. Bolk, J. Ireland, P.C. Sabeti, D.J. Richter, T. Lavery, R. Kouyoumjian, S.F. Farhadian, R. Ward, and E. S. Lander. 2001. Linkage disequilibrium in the human genome. Nature 411:199-204.

46.Riley, J.H., C.J. Allan, E. Lai, and A. Roses. 2000. The use of single nucleotide polymorphisms in the isolation of common disease genes. Pharmacogenomics 1:39-47.

47.Rioux, J.D., M.S. Silverberg, M.J. Daly, A.H. Steinhart, R.S. McLeod, A.M. Griffiths, T. Green, T.S. Brettin, et al. 2000. Genomewide search in Canadian families with inflammatory bowel disease reveals two novel susceptibility loci. Am. J. Hum. Genet. 66:1863-1870.

48.Rioux, J.D., M.J. Daly, M.S. Silverberg, K. Lindblad, H. Steinhart, Z. Cohen, T. Delmonte, K. Kocher, et al. 2001. Genetic variation in the 5q31 cytokine gene cluster confers susceptibility to Crohn disease. Nat. Genet. 29:223-228.

49.Saiki, R.K., P.S. Walsh, C.H. Levenson, and H.A. Erlich. 1989. Genetic analysis of amplified DNA with immobilized sequence-specific oligonucleotide probes. Proc. Natl. Acad. Sci. USA 86:6230-6234.

50.Sauer, S., D.H. Gelfand, F. Boussicault, K. Bauer, F. Reichert, and I.G. Gut. 2002. Facile method for automated genotyping of single nucleotide polymorphisms by mass spectrometry. Nucleic Acids Res. 30:e22 .

51.Shi, M.M. 2001. Enabling large-scale pharmacogenetic studies by highthroughput mutation detection and genotyping technologies. Clin. Chem. 47:164-172.

52.Stoerker, J., J.D. Mayo, C.N. Tetzlaff, D.A. Sarracino, I. Schwope, and C. Richert. 2000. Rapid genotyping by MALDI-monitored nuclease selection from probe libraries. Nat. Biotechnol. 18:1213-1216.

53.Syvanen, A.C. 1999. From gels to chips: "minisequencing" primer extension for analysis of point mutations and single nucleotide polymorphisms. Hum. Mutat. 13:1-10

54.Tyagi, S., D.P. Bratu, and F.R. Kramer. 1998. Multicolor molecular beacons for allele discrimination. Nat. Biotechnol. 16:49-53.

55.Venter, J.C., M.D. Adams, E.W. Myers, P.W. Li, R.J. Mural, G.G. Sutton, H.O. Smith, M. Yandell, et al. 2001. The sequence of the human genome. Science 291:1304-1351

56.Watson, J. and F. Crick. 1953. Molecular structure of nucleic acids. Nature 171:737-738.

57.Ye, F., M.-S. Li, J.D. Taylor, Q. Nguyan, H.M. Colton, W.M. Casey, M. Wagner, M.P. Weiner, and J. Chen. 2001. Fluorescent microspere-based multiplex assay for human single nucleotide polymorphism analysis and bacterial identification. Hum. Mutat. Res. 17:305-316.

\section{Address correspondence to:}

Dr. Michael P. Weiner

454 Corporation

20 Commercial Street

Branford, CT 06405, USA

e-mail:mweiner@454.com 